\title{
ON BOUNDED pO-SEMIGROUPS
}

\author{
ZAHAVA SHMUELY
}

\begin{abstract}
ABstkact. The bounded po-semigroup $S$ is investigated by studying its increasing elements $u\left(\leqslant u^{2}\right)$ and decreasing elements $v\left(\geqslant v^{2}\right)$. In particular, in $S, 01\left(=0^{n} 1^{m}\right), 10\left(=1^{n} 0^{m}\right), 010$ and 101 are all idempotents and $010=01 \wedge_{E} 10,101=10 \vee_{E} 01, E$ the set of idempotents of $S$ ordered as a subset of $S$. In $S, 0 a 1=01$ and $1 a 0=10$ holds for each $a \in S$. Consequently, $S$ has a zero element $z$ iff $01=10$ and in that case $z=01$. $S$ cannot be cancellative unless it is trivial. $J_{0}=S 10 S \subseteq S$ is the kernel of $S$ and consists of all (idempotents) $a \in S$ satisfying $a S a=a$. Thus when $S$ is a (zero) simple bounded po-semigroup then $a S a=\{a, z\}$ and either $a^{2}=a$ or $a^{2}=z$ for each $a \in S$. When $S=X^{X}$, the po-semigroup of isotone maps $f$ on the bounded poset $X$, then $J_{0}$ consists of all constant maps on $X$, hence $J_{0} \simeq X$. The following generalization of Tarski's fixed point theorem is obtained: Let $S$ be a complete (lattice and a) po-semigroup and let $s \in S$ be given. Then the set $E_{s}\left(J_{s}\right)$ of all elements $x_{0} \in E\left(\in J_{0}\right.$ resp.) satisfying $s x_{0}=x_{0} s=x_{0}$ is a nonempty complete lattice when ordered as a subset of $S$.
\end{abstract}

1. Let $S$ denote a partially ordered (po) semigroup [1], [3]. Thus $S$ is a semigroup endowed with a partial order $\leqslant$, such that $a \leqslant b \in S$ implies $a c \leqslant b c$ and $c a \leqslant c b$ for each $c \in S$. $S$ is bounded if $S$ contains universal bounds 0,1 such that $0 \leqslant s \leqslant 1$ for each $s \in S$. If $S$ is a po-semigroup which is a complete lattice with respect to $\leqslant$ we say that $S$ is a complete posemigroup. An element $z(i)$ satisfying $z s=s z=z$ (is $=s i=s$ resp.) for each $s \in S$ is a zero (identity resp.) element of the semigroup $S$ [2]. In general, 0, 1 must not be interchanged with $z$ and $i$. Thus, e.g., let $S=X^{X}$ [1], be the posemigroup of isotone maps $f: X \rightarrow X, X$ a bounded poset. The semigroup operation in $X^{X}$ is function composition and the order is the pointwise partial order. In $X^{X}$ we clearly have $01=0,10=1$.

In this paper we study algebraic properties of a po-semigroup $S$. Two classes of elements, more general than the classes of positive and negative elements [3, p. 154], usually studied in po-semigroups, are introduced and shown to be of special significance. Thus $u \in S$ is increasing if $u \leqslant u^{2}$, and $v \in S$ is decreasing if $v^{2} \leqslant v$. Obviously, $e$ is both increasing and decreasing iff $e$ is idempotent, namely $e=e^{2}$. Let $U, V \subseteq S$ denote the sets of increasing (decreasing resp.) elements of $S . U, V$ and $E=U \cap V$ are ordered as subsets of $S$. Increasing and decreasing elements of $X^{X}$ are treated in [6] and shown to form natural extensions of closure and anticlosure operators. As noted in $[6, \S 7]$, it is the combined increasing-decreasing character of the identity $i$

Received by the editors May 4, 1975.

AMS (MOS) subject classifications (1970). Primary 06A50, $20 \mathrm{M} 10$.

(1) American Mathematical Society 1976 
which is mainly responsible to the peculiar properties of Galois connections [5], [1] between two posets.

The existence in a po-semigroup $S$ of elements $u, v$ such that $u \in U, v \in V$ and $u \leqslant v$ (which is the case, e.g., for 0,1 when $S$ is bounded since $\left.0 \leqslant 0^{2} \leqslant 1^{2} \leqslant 1\right)$ gives rise to results of basic importance. Thus $u v, v u, u v u$ and $v u v$ are idempotents with $u v u=u v \wedge_{E} u v, v u v=v u \vee_{E} u v$. The structure of the subsemigroup $S^{*}$ generated by $\{u, v\}$ is determined. For a bounded posemigroup $S$ we have: $S$ contains a zero $z$ iff $01=10$ holds, and in that case $z=01$. $S$ cannot be cancellative unless it is trivial. $S$ has a nonempty kernel $J_{0}=S 10 S$, which is the minimal ideal of $S$ and $x \in J_{0}$ iff $x=x^{2}=x S x$. When $S=X^{X}, J_{0}$ consists of all constant maps, hence $J_{0} \approx X$. A characterization of (zero) simple bounded po-semigroups follows. Thus, e.g., when $S$ is zero-simple, either $z=1$ or $z=0$, and for each $x \in S$ either $x^{2}=x$ or $x^{2}=z$ and $x S x=\{x, z\}$ for $x \neq z$. Consequently a (zero) simple bounded posemigroup is completely (zero) simple.

It is interesting to note that an analogue of Tarski's fixed point theorem [7], [1, p. 115], holds in an arbitrary complete po-semigroup $S$. Thus for each $s \in S$ the set of idempotent elements $x_{0}(\in E) \in J_{0}$ satisfying $x_{0} s=s x_{0}$ $=x_{0}$ is a nonempty complete lattice when ordered as a subset of $S$. When applied to $S=X^{X}$ this is precisely Tarski's theorem.

2. We deal here with some basic algebraic properties of po-semigroups. At first we have:

Lemma 1.2. Let $v^{2} \leqslant v \in S$ where $S$ is a po-semigroup, and let $x \leqslant v$. Then: (i) $x v \leqslant v, v x \leqslant v$; (ii) both $v x$ and $x v$ are decreasing elements.

Proof. (i) follows by $x v \leqslant v^{2} \leqslant v$ and $v x \leqslant v^{2} \leqslant v$. To prove (ii) note that $(x v)^{2} \leqslant x v^{3} \leqslant x v^{2} \leqslant x v .(v x)^{2} \leqslant v x$ follows similarly.

Dually we get:

LEMma $1^{*}$.2. Let $u \leqslant u^{2} \in S$ where $S$ is a po-semigroup, and let $y \geqslant u$. Then: (i) $y u \geqslant u, u y \geqslant u$; (ii) both uy and yu are increasing elements.

COROLlaRY 1.2. If $v \in S$ is decreasing, then ( $v$ ], the principal (order) ideal generated by $v$ is a convex po-subsemigroup of $S$. If $u \in S$ is increasing, then $[u$ ), the principal dual (order) ideal generated by $u$ is a convex po-subsemigroup of $S$.

If in a po-semigroup $S$ a pair $(u, v)$ of elements is given such that $u$ in increasing, $v$ is decreasing and $u \leqslant v$ then the chain of inclusions

$$
u \leqslant u^{2} \leqslant \cdots \leqslant u^{n} \leqslant \cdots \leqslant v^{n} \leqslant \cdots \leqslant v^{2} \leqslant v
$$

is obtained. Note that in a po-group the existence of such a pair $(u, v)$ is impossible unless $u=v=i$. The following theorem plays a central role in what follows.

THEOREM 1.2. Let $S$ be a po-semigroup and let $u, v \in S$ be given such that $u$ is increasing, $v$ is decreasing and $u \leqslant v$. Then: (i) for each $x \in[u, v]$ we have $u x v=u v, v x u=v u$; (ii) $u^{n} v^{m}=u v, v^{n} u^{m}=v u$ for $n, m \geqslant 1$; (iii) the elements $u v, v u$, uvu, and $v u v$ are idempotents; (iv) $u v u=u v \wedge_{E} v u=u v \wedge_{U} v u$, and $v u v=v u \vee_{E} u v=v u \vee_{V} u v$. 
Proof. If $u \leqslant x \leqslant v$ then $u v \leqslant u^{2} v \leqslant u x v \leqslant u v^{2} \leqslant u v$ and $u x v=u v$. Similarly, we get $v x u=v u$ and (i) follows. (ii) is a special case of (i) since $u^{k} v^{l}, v^{k} u^{l} \in[u, v]$ for $k, l>0$. To prove (iii) note that by Lemmas 1.2(ii), $1^{*}$.2(ii) the elements $u v, v u$ are both increasing and decreasing, hence are idempotent. Since $u \leqslant u^{2} \leqslant(u v)^{2}=u v, v u=(v u)^{2} \leqslant v^{2} \leqslant v$, the last mentioned result applied again yields that both $u v u$ and $v u v$ are idempotent. To prove (iv) note that $u v u \leqslant u v, v u$. Now if $t \leqslant t^{2} \in S$ satisfies $t \leqslant u v, t \leqslant v u$ then obviously $t \leqslant t^{2} \leqslant u v v u=u v u$ by (ii) and $u v u=u v \wedge_{E} v u=u v \wedge_{U} v u$ follows. The second part of (iv) follows similarly.

COROllaRY 2.2. If a po-semigroup $S$ contains elements $u, v$ such that $u \leqslant u^{2} \leqslant v^{2} \leqslant v$ then $E \neq \varnothing$.

Note that under the conditions of Theorem 1.2 it acutally follows that if $u \leqslant x \leqslant y \leqslant v$ and if $u_{1}$ denotes either $u x$ or $x u$ while $v_{1}$ denotes either $v y$ or $y v$ then both $u_{1} v_{1}$ and $v_{1} u_{1}$ are idempotents with $u_{1} v_{1} u_{1}=u_{1} v_{1} \wedge_{E} v_{1} u_{1}$, $v_{1} u_{1} v_{1}=v_{1} u_{1} \vee_{E} u_{1} v_{1}$.

COROLlary 3.2. Let $S$ be a po-semigroup and let $u, v \in S$ satisfy $u \leqslant u^{2}$ $\leqslant v^{2} \leqslant v$. Then $\hat{S}$, the subsemigroup generated by $\{u, v\}$ is an order-and semigroup-epimorphic image of $S^{*}$, the "free" po-semigroup generated by $u$ and $v$ where

$$
S^{*}=\left\{u^{n}\right\}_{n=1,2, \ldots} \cup\left\{v^{n}\right\}_{n=1,2, \ldots} \cup\{u v u, u v, v u, v u v\} .
$$

Notice that the last four elements in (2) form an idempotent po-subsemigroup. $S^{*}$ is given in Figure 1 with multiplication given by Theorem 1(ii).

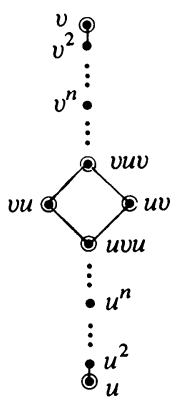

FIGURE 1

Theorem 1 and Corollary 3 hold, in particular, when either $u, v$ or both $u$ and $v$ are idempotents. Thus if $u=u^{2} \leqslant v=v^{2}$ then $S^{*}$, the po-subsemigroup generated by $u$ and $v$, is an idempotent semigroup consisting of the six encircled elements in Figure 1. Clearly when $S$ is linearly ordered then $S^{*}$ consists of at most four elements (this is the case in [4] where $S$ is a linearly ordered idempotent semigroup). We conclude this section with

Corollary 4.2. Let $S$ be a po-semigroup. Then $S \subseteq \hat{S}$ is a convex bounded posubsemigroup of $S$ iff $\hat{S}=[u, v]$ for some $u, v \in S$ such that $u \leqslant u^{2} \leqslant v^{2} \leqslant v$. 
3. In this section we apply the results of $\$ 2$ to the bounded po-semigroup $S$. Since $0 \leqslant 0^{2} \leqslant 1^{2} \leqslant 1$, it follows by Theorem 1.2 that the elements 01,10 , $010=01 \wedge_{E} 10$ and $101=10 \vee_{E} 01$ are all idempotent, and for each $x \in S$

$$
0 x 1=01, \quad 1 x 0=10 .
$$

The following lemma is well known for po-groups.

LEMMA 1.3. A cancellative po-semigroup cannot be bounded unless it is trivial.

Proof. Assuming $S$ is both bounded and cancellative we get by (3) that $0^{3} 1=0^{2} 1,01^{3}=01^{2}$ implying that $0=01=1$.

The question of the existence of a zero element in a bounded po-semigroup is settled by

THEOREM 1.3. Let $S$ be a bounded po-semigroup. Then $S$ has a zero element $z$ iff $01=10$, and in that case $z=01$.

Proof. If $z \in S$, then $01 \leqslant z 1=z=0 z \leqslant 01$ and so $z=01$, while $10 \leqslant 1 z=z=z 0 \leqslant 10$ implies $z=10$. Conversely, if $01=10$ holds, then for each $x \in S, 01=0^{2} 1 \leqslant x 01 \leqslant 101=01^{2}=01$, hence $x 01=01.01 x$ $=01$ follows similarly and $01=z$ is the unique, two-sided zero element of $S$.

COROLlaRY 1.3. A commutative bounded po-semigroup contains a zero element.

The following can easily be proved using (3).

LEMMA 2.3. Let e denote any of the idempotents $01,10,101$ or 010 in a bounded po-semigroup $S$. Then: (i) $e$ is a primitive idempotent satisfying $e S e=e$; (ii) if $a=$ xey for some $x, y \in S$ then $a=a^{2}=a S a$.

$J \subseteq S$ is a (semigroup) ideal of $S$ if $J S \subseteq J, S J \subseteq J$. Let $J_{0}$, the kernel of $S$, denote the intersection of all ideals of $S$. $S$ is (zero) simple [2] if $S$ contains no proper ideal (except $\{z\}$ when $z \in S$ ).

THEOREM 2.3. Let $S$ be a bounded semigroup. Then $J_{0}$, the kernel of $S$, is nonempty and consists of all elements $x \in S$ satisfying $x=x^{2}=x S x . J_{0}$ $=S 01 S=S x S$ for each $x \in J_{0}$.

Proof. If $J$ is a nonempty ideal of $S$ then $01=0 J 1 \subseteq S J S \subseteq J$ (see (3)). Thus $S 01 S \subseteq J$ for each ideal $J$ and $J_{0}=S 01 S$. By Lemma 2.3 we get that each $x \in J_{0}$ satisfies $x=x^{2}=x S x$, and obviously $J_{0} \subseteq S x S \subseteq S 01 S=J_{0}$. Conversely if $x=x S x$ then $x=x 01 x \in S 01 S=J_{0}$ which completes the proof.

Notice that by Theorem 1.3, $J_{0}=\{z\}$ iff $01=10$.

Corollary 2.3. Let $S=X^{X}$ where $X$ is a bounded poset. Then $J_{0} \approx X$ and consists of all constant maps on $X$.

Proof. In $S, 0 f=0$ holds for each $f \in S$. Thus $J_{0}=S 01 S=S 0$ and $g \in J_{0}$ iff $g=f 0$, i.e., $g(x)=f(0)$ for each $x \in X$. Hence $J_{0}$ consists of constant maps. Moreover, since $g=g S g$ holds for each constant map $g \in S$ the theorem follows using Theorem 2.3. 
In case $S$ is a complete po-semigroup we get:

THEOREM 3.3. If $S$ is a complete po-semigroup then $J_{0}$, the kernel of $S$, is a complete lattice in the order induced by $S$.

Proof. Let $\left\{x_{j}\right\} \subseteq J_{0}$ be given. If $y=\vee x_{j}$ then $y_{0}=y 01 y \geqslant x_{j} 01 x_{j}=x_{j}$ for each $j$, so $y_{0} \in J_{0}$ and $y_{0} \geqslant \vee x_{j}=y$. If for some $x \in J_{0}, x \geqslant \vee x_{j}=y$, then $x=x 01 x \geqslant y 01 y=y_{0}$ follows and so $y_{0}=\bigvee_{J_{0}} x_{j}$. The existence of $\wedge_{J_{0}} x_{j}$ is proved similarly.

Turning now to bounded po-semigroups which are (zero) simple we get using Theorem 2.3:

THEOREM 4.3. Let $S$ be a bounded po-semigroup without zero. Then $S$ is simple iff $S$ is an idempotent semigroup with $x S x=x$ for each $x \in S$, that is, iff $S$ is a rectangular band.

Let us now assume that $S$ has a zero element $(z=01=10)$ and is zerosimple. Assuming $z \neq 1$ it follows (see [2, Lemma 2.28]) that $S=S 1 S$ hence $0=x 1 y$ for some $x, y \in S$ and so $0=x 1 y \geqslant 010=z \geqslant 0$ implying $z=0$. Similarly $z \neq 0$ would imply $z=1$. In both cases we have

THEOREM 5.3. Let $S$ be a zero-simple bounded po-semigroup. Then for each $x \in S$ either $x^{2}=x$ or $x^{2}=z$ and $x S x=\{x, z\}$.

Proof. We can assume $z=0=10=01$ and $z \neq 1$. Since $S=S x S$ for $x \neq z$,

$$
1 x 1=1
$$

is obvious. By $S=S 1 S$ we get that each $(z \neq) x \in S$ satisfies $x=a 1 b$ for some $a, b \in S$. Consequently $x^{2}=a 1 b a 1 b$ equals either $x$ (if $b a \neq z$ ) or $z$ (if $b a=z) . x S x=a 1 b S a 1 b=\{x, z\}$ follows by observing that $b S a \neq\{z\}$ when $b, a$ $\neq z[2$, Chapter 2] and by $(*)$.

COROLlaRY 3.3. A (zero) simple bounded po-semigroup $S$ is completely (zero) simple.

4. Here we show that analogues of Tarski's fixed point theorem hold in any complete po-semigroup $S$. If $x_{0} s=s x_{0}=x_{0}$ holds for some $s, x_{0} \in S$ we say that $x_{0}$ is fixed by $s$. We now state

THEOREM 1.4. Let $S$ be a complete po-semigroup and let $s \in S$ be given. Then there exists an idempotent $x_{0} \in S$ which is fixed by $s$. Moreover, $E_{s}$, the set of idempotents fixed by $s$ is a complete lattice when ordered as a subset of $S$.

This theorem will follow by

Lemma 1.4. Let $S$ be a complete po-semigroup and let $s \in S$ be given. If $m \in S$ is increasing and $s m \geqslant m, m s \geqslant m$ then there exists $y_{0} \in S$ such that (i) $m \leqslant y_{0}$, (ii) $y_{0}=y_{0}^{2}$, (iii) $y_{0}$ is fixed by $s$ and (iv) $y_{0}$ is the least element satisfying (i), (ii), and (iii). 
Proof. Let $X_{s}$ denote the set of decreasing elements $x_{j} \in S$ satisfying $m \leqslant x_{j}, s x_{j} \leqslant x_{j}, x_{j} s \leqslant x_{j} . X_{s} \neq \varnothing$ since $1 \in X_{s}$. Letting $y_{0}=\wedge x_{j}$ we have

$$
y_{0}^{2}=\left(\wedge x_{j}\right)\left(\wedge x_{j}\right) \leqslant \wedge x_{j}^{2} \leqslant \wedge x_{j}=y_{0} .
$$

Obviously

$$
m \leqslant \wedge x_{j}=y_{0}, \quad s y_{0}=s\left(\wedge x_{j}\right) \leqslant \wedge s x_{j} \leqslant \wedge x_{j}=y_{0},
$$

and $y_{0} s \leqslant y_{0}$ follows similarly, hence $y_{0} \in X_{s}$. By $\left(y_{0}^{2}\right)^{2} \leqslant y_{0}^{2}$ and $m \leqslant m^{2}$ $\leqslant y_{0}^{2}$ together with $s y_{0}^{2} \leqslant y_{0}^{2}$ and $y_{0}^{2} s \leqslant y_{0}^{2}$ we have $y_{0}^{2} \in X_{s}$. Thus $y_{0} \leqslant y_{0}^{2}$, and $y_{0}=y_{0}^{2} \in E$ follows. Obviously $m \leqslant m s \leqslant y_{0} s$, and $\left(y_{0} s\right)\left(y_{0} s\right) \leqslant y_{0}^{2} s$ $=y_{0} s$. Thus $y_{0} s$ is decreasing. By $s\left(y_{0} s\right)=\left(s y_{0}\right) s \leqslant y_{0} s$ together with $\left(y_{0} s\right) s \leqslant y_{0} s$ one gets that $y_{0} s \in X_{s}$ and so $y_{0} \leqslant y_{0} s . y_{0} s=y_{0}$ follows and $s y_{0}=y_{0}$ can be shown similarly. Consequently $y_{0}$ was shown to satisfy (i), (ii), (iii), and is by definition the least element having these properties.

Proof of Theorem 1.4. Since $0 \leqslant 0^{2}$ with $0 \leqslant s 0,0 \leqslant 0 s$, Lemma 1.4 implies the existence of a minimal idempotent $y_{0} \geqslant 0$ which is fixed by $s$. Obviously, $y_{0}=0_{E_{s}}$. For any set $\left\{y_{j}\right\} \subseteq E_{s}$ put $m=\vee y_{j}$. The fact that $m$ is increasing with $m \leqslant m s, m \leqslant s m$ is easily checked. Lemma 1.4 applied again yields the existence of an idempotent $y_{0}^{*}$ fixed by $s$ such that $y_{0}^{*}=\bigvee_{E_{s}} y_{j}$. Thus $E_{s}$ is a complete lattice.

Tarski's theorem can be even better "approximated" (Corollary 2.3) and actually generalized by

THEOREM 2.4. Under the conditions of Theorem 1.4 the set $J_{s} \subseteq E_{s}$ of elements $y_{0} \in J_{0}$ fixed by $s$ is a nonempty complete lattice in the order induced by $S$.

Proof. Obviously (Theorem 2.3) $J_{0} \subseteq E$ and for each $x \in E_{s}, x_{0}=x 01 x$ $\in J_{0}$ and $x_{0}$ is fixed by $s$. Thus $J_{s} \neq \varnothing$. For any set $\left\{x_{j}\right\} \subseteq J_{s}$ let $\hat{x}=\wedge_{E_{s}} x_{j}$ (Theorem 1.4). Then as in Theorem 3.3 one can easily show that $\hat{x} 01 \hat{x}$ $=\wedge_{J_{s}} x_{j}$. The existence of $\bigvee_{J_{s}} x_{j}$ is proved analogously.

By similar methods another of Tarski's theorems [7] can be generalized:

THEOREM 3.4 Let $S$ be a complete po-semigroup and let $s_{1} s_{2}=s_{2} s_{1}$ for some $s_{1}, s_{2} \in S$. Then $E_{s_{1}} \cap E_{s_{2}}$ is a nonempty complete lattice.

As an application of Theorem 2.4, we state

CoROllary 1.4. Let $R$ be a ring and let $I_{0} \subseteq R$ be a given (two-sided) ideal. Then the set of all ideals $I \subseteq R$ satisfying $I_{0} I=I I_{0}=I=I^{2}$ is a nonempty complete lattice when ordered by inclusion.

\section{REFERENCES}

1. G. Birkhoff, Lattice theory, 3rd ed., Amer. Math. Soc. Colloq. Publ., vol. 25, Amer. Math. Soc., Providence, R.I., 1967. MR 37.

2. A. H. Clifford and G. B. Preston, The algebraic theory of semigroups, Vol. I, Math. Surveys, no. 7, Amer. Math. Soc., Providence, R.I., 1961. MR 24 \# A2627.

3. L. Fuchs, Partially ordered algebraic systems, Pergamon Press, New York; Addison-Wesley, Reading, Mass., 1963. MR 30 \#2090. 
4. T. Merlier, Nouvelles propriétés algébriques des demi-groupes idempotents totalement ordonnés, C.R. Acad. Sci. Paris Sér. A.-B 277 (1973), A451-A452. MR 48 \# 3832.

5. O. Ore, Galois connexions, Trans. Amer. Math. Soc. 55 (1944), 493-513. MR 6, 36.

6. Z. Shmuely, Increasing and decreasing operators on complete lattices, J. Combinatorial Theory Ser. A (to appear).

7. A. Tarski, $A$ lattice-theoretical fixpoint theorem and its applications. Pacific J. Math. 5 (1955), 285-309. MR 17, 574.

Department of Mathematics, Technion-Israel Institute of Technology, Haifa, Israel 